\title{
LINEAR DIFFUSION WITH STATIONARY SWITCHING REGIME
}

\author{
Xavier Guyon ${ }^{1}$, Serge IovlefF ${ }^{2}$ and Jian-Feng YaO ${ }^{3}$
}

\begin{abstract}
Let $Y$ be a Ornstein-Uhlenbeck diffusion governed by a stationary and ergodic process $X: \mathrm{d} Y_{t}=a\left(X_{t}\right) Y_{t} \mathrm{~d} t+\sigma\left(X_{t}\right) \mathrm{d} W_{t}, Y_{0}=y_{0}$. We establish that under the condition $\alpha=E_{\mu}\left(a\left(X_{0}\right)\right)<0$ with $\mu$ the stationary distribution of the regime process $X$, the diffusion $Y$ is ergodic. We also consider conditions for the existence of moments for the invariant law of $Y$ when $X$ is a Markov jump process having a finite number of states. Using results on random difference equations on one hand and the fact that conditionally to $X, Y$ is Gaussian on the other hand, we give such a condition for the existence of the moment of order $s \geq 0$. Actually we recover in this case a result that Basak et al. [J. Math. Anal. Appl. 202 (1996) 604-622] have established using the theory of stochastic control of linear systems.
\end{abstract}

Mathematics Subject Classification. 60J60, 60J75.

Received February 4, 2003. Revised March 17, 2003.

\section{INTRODUCTION}

The discrete time models $Y=\left(Y_{n}, n \in \mathbf{N}\right)$ governed by a switching process $X=\left(X_{n}, n \in \mathbf{N}\right)$ fit well to the situations where an autonomous process $X$ is responsible for the dynamic (or regime) of $Y$. These models are parsimonious with regard to the number of parameters, and extend significantly the case of a single regime. Among them, the Markov switching ARMA models are the most popular. Their use in econometric modeling is due to Hamilton $[7,8]$. Their statistical study ( $c f$. for example $[8-10,15])$ has preceded their probabilistic study. The ergodicity has been examined by Francq and Roussignol [6] and Yao and Attali [17]. In this last work, the authors give:

(i) conditions for the stability of a non-linear AR process $Y$ under the Markovian switching $X$;

(ii) conditions of existence of a moment of order $s \geq 0$ for the law of $Y$.

These two results, obtained under sub-linearity or Lipschitz conditions for the auto-regression function, are preliminary tools for any estimation theory.

Our objective is to establish results similar to (i) and (ii) for a Ornstein-Uhlenbeck diffusion (denoted O.U.) $Y=\left(Y_{t}, t \geq 0\right)$ with switching $X=\left(X_{t}, t>0\right)$. We obtain a stability condition (i) for $Y$ under general switching $X$ process which is assumed stationary and ergodic. The question (ii) has been studied in Basak et al. [1] in the case of a Markovian switching $X$ having finite number of states. Their approach relies on the theory of stochastic control of linear systems ( $c f$. Mariton [14], Ji and Chizeck [12]). Our approach to these questions \footnotetext{
existence of moments.

1 SAMOS, Université Paris 1, France; e-mail: Xavier.Guyon@univ-paris1.fr

2 LMA, Université de Lille 1, France; e-mail: serge.iovleff@univ-lille1.fr

3 IRMAR, Université de Rennes 1, France; e-mail: Jian-Feng. Yao@univ-rennes1.fr
}

Keywords and phrases. Ornstein-Uhlenbeck diffusion, Markov switching, jump process, random difference equations, ergodicity, 
is different: we first investigate the ergodicity for the family of discretizations $Y^{(\delta)}=\left(Y_{n \delta}, n \in \mathbf{N}\right)$ using the theory of random difference equations ( $c f$. e.g. [3]). The ergodicity of the process $Y$ itself is then obtained by the approximation of $Y$ by the discretizations. Here we also used the conditionally Gaussian character of $Y$. For conditions ensuring the existence of moments, the study of the discretizations is also helpful because they have the same stationary distribution as the original $Y$. Some simple manipulations show that our result to Question (ii) is equivalent to the one given by [1].

The linear diffusion model with switching is presented in Section 1. In Section 2, the condition (i) for the ergodicity of $Y$ is established when the switching $X$ is stationary. Then in Section 3, assuming the particular case where $X$ is a Markov jump process having a finite number of states, we establish conditions for the existence of a moment of order $s \geq 0$ for the invariant law of $Y$. Some simulation is given at the end to illustrate the results.

\section{LINEAR DIFFUSION WITH STATIONARY SWITCHING}

We will say a continuous time process $S=\left(S_{t}\right)_{t>0}$ is ergodic if there exists a probability measure $\nu$ such that when $t \rightarrow \infty$, the law of $S_{t}$ converges weakly to $\nu$ independently of the initial condition $S_{0}$. The distribution $\nu$ is then the limit law of $S$. When $S$ is a Markov process, $\nu$ is its unique invariant law. Note that this definition of ergodicity is specific to our context for the ease of statements.

We define a diffusion $Y$ with a switching $X$ in two steps. First we take a process $X=\left(X_{t}\right)_{t \geq 0}$, called the switching process. We will always suppose in the following that $X$ is defined on a probability space $(\Omega, \mathcal{A}, Q)$, real-valued, stationary and ergodic with limit law $\mu$.

Let $W=\left(W_{t}\right)_{t \geq 0}$ be a standard Brownian motion defined on a probability space $\left(\Theta, \mathcal{B}, Q^{\prime}\right), \mathcal{F}=\left(\mathcal{F}_{t}\right)$ the filtration of the motion. We will consider the product space $\left(\Omega \times \Theta, \mathcal{A} \times \mathcal{B}, Q \otimes Q^{\prime}\right), \mathbb{P}=Q \otimes Q^{\prime}$ and $\mathbb{E}$ the associated expectation. Conditionally to $X, Y=\left(Y_{t}\right)_{t \geq 0}$ is a real-valued diffusion process, defined, for each $\omega \in \Omega$, by:

(1) $Y_{0}$ is a random variable defined on $\left(\Theta, \mathcal{B}, Q^{\prime}\right), \mathcal{F}_{0}$-measurable;

(2) $Y$ is solution of the linear SDE

$$
\mathrm{d} Y_{t}=a\left(X_{t}\right) Y_{t} \mathrm{~d} t+\sigma\left(X_{t}\right) \mathrm{d} W_{t}, \quad t \geq 0 .
$$

Thus $\left(Y_{t}\right)$ is a linear diffusion driven by a "exogenous" process $\left(X_{t}\right)$. Here $a$ and $\sigma$ are two real valued measurable functions. The existence and the uniqueness of a strong solution for equation (1) is guaranteed under the following condition (see [13], Sect. 5.6 or [16]):

[S] $Q$-a.s., $t \mapsto a\left(X_{t}(\omega)\right)$ and $t \mapsto \sigma\left(X_{t}(\omega)\right)$ are locally bounded.

This condition will be assumed satisfied throughout the paper.

For $0 \leq s \leq t$, let

$$
\Phi(s, t)=\Phi_{s, t}(\omega)=\exp \int_{s}^{t} a\left(X_{u}\right) \mathrm{d} u .
$$

The process $Y$ has the representation [13]:

$$
Y_{t}=Y_{t}(\omega)=\Phi(0, t)\left[Y_{0}+\int_{0}^{t} \Phi(0, u)^{-1} \sigma\left(X_{u}\right) \mathrm{d} W_{u}\right]
$$

and for $0 \leq s \leq t, Y$ satisfies the recursion equation:

$$
\begin{aligned}
Y_{t} & =\Phi(s, t)\left[Y_{s}+\int_{s}^{t} \Phi(s, u)^{-1} \sigma\left(X_{u}\right) \mathrm{d} W_{u}\right] \\
& =\Phi(s, t) Y_{s}+\int_{s}^{t}\left[\exp \int_{u}^{t} a\left(X_{v}\right) \mathrm{d} v\right] \sigma\left(X_{u}\right) \mathrm{d} W_{u} .
\end{aligned}
$$


It is useful to rewrite this recursion as

$$
Y_{t}(\omega)=\Phi_{s, t}(\omega) Y_{s}(\omega)+V_{s, t}(\omega)^{1 / 2} \xi_{s, t}
$$

where $\xi_{s, t}$ is a standard Gaussian variable, function of $\left(W_{u}, s \leq u \leq t\right)$ and

$$
V_{s, t}(\omega)=\int_{s}^{t} \exp \left[2 \int_{u}^{t} a\left(X_{v}\right) \mathrm{d} v\right] \sigma^{2}\left(X_{u}\right) \mathrm{d} u
$$

For $\delta>0$, we will call discretization at step size $\delta$ of $Y$ the discrete time process $Y^{(\delta)}=\left(Y_{n \delta}\right)_{n}$ where $n \in \mathbb{N}$. Our study of $Y$ is based on the investigations of these discretizations $\left(Y^{(\delta)}\right)$.

Let us mention that under Assumption [S], the regime process has the following property: as $\delta \rightarrow 0$,

$$
\int_{0}^{\delta} \sigma^{2}\left(X_{s}\right) \mathrm{d} s \rightarrow 0, \quad \text { almost surely }
$$

as we have a.s. $\int_{0}^{t} \sigma^{2}\left(X_{s}\right) \mathrm{d} s<\infty$ for all $t>0$. The claim is then a consequence of Lebesgue's dominated convergence theorem.

\section{ERgodicity of $Y$ AND EXISTENCE OF A STATIONARY SOLUTION}

\subsection{Ergodicity of the discretized process $Y^{(\delta)}$}

In this section, we fix $\delta>0$ and consider the discretization $Y^{(\delta)}$. According to equation $(2)$, for $n \geq 0$,

$$
Y_{(n+1) \delta}(\omega)=\Phi_{n+1}(\omega) Y_{n \delta}(\omega)+V_{n+1}(\omega)^{1 / 2} \xi_{n+1},
$$

with

$$
\begin{aligned}
\Phi_{n+1}(\omega) & =\exp \int_{n \delta}^{(n+1) \delta} a\left(X_{u}(\omega)\right) \mathrm{d} u \\
V_{n+1}(\omega) & =\int_{n \delta}^{(n+1) \delta} \exp \left[2 \int_{u}^{(n+1) \delta} a\left(X_{v}(\omega)\right) \mathrm{d} v\right] \sigma^{2}\left(X_{u}(\omega)\right) \mathrm{d} u
\end{aligned}
$$

where $\left(\xi_{n}\right)$ is a i.i.d. sequence of standard Gaussian variables defined on $\left(\Theta, \mathcal{B}, Q^{\prime}\right)$.

The equation (4) defines an $\operatorname{AR}(1)$ model with random coefficients. As the coefficients $\left(\Phi_{n}, V_{n}^{1 / 2} \xi_{n}\right)$ are stationary, we can then extend (4) on $\mathbb{Z}$ by standard construction. Let us define for $x>0, \log ^{+} x=\max (0, \log x)$. This function has the properties: $\log ^{+}(x y) \leq \log ^{+} x+\log ^{+} y$ and $\log ^{+} x^{a}=a \log ^{+} x$ for any positive $x, y$ and $a$. Recall that $\mu$ is the stationary law of $X$.

Proposition 1. Assume that the measurable functions a and $\sigma$ verify the following conditions:

(1) $\int|a(x)| \mu(\mathrm{d} x)<\infty$ and $\alpha:=\int \mu(\mathrm{d} x) a(x)<0$;

(2) for some $\varepsilon>0, \mathbb{E}\left[\log ^{+} \int_{0}^{\varepsilon} \sigma^{2}\left(X_{u}\right) \mathrm{d} u\right]<\infty$.

Then,

(i) there exists an unique stationary solution $\left(\tilde{Y}_{n \delta}\right)$ satisfying on $\mathbb{Z}$ the equation (4) and given by

$$
\tilde{Y}_{n \delta}=\sum_{k=0}^{\infty} \Phi_{n} \Phi_{n-1} \cdots \Phi_{n-k+1} V_{n-k}^{1 / 2} \xi_{n-k}, \quad n \in \mathbb{Z}
$$


(ii) for any solution $Y^{(\delta)}$ of equation (4) starting with a arbitrary condition $Y_{0}$, we have a.s.

$$
\limsup _{n \rightarrow \infty} \frac{1}{n} \log \left|Y_{n \delta}-\tilde{Y}_{n \delta}\right| \leq \alpha \delta<0
$$

Proof. Part (i). It is a consequence of Theorem 1 of Brandt [3] for which we verify the conditions of application:
(a) $\mathbb{E} \log ^{+}\left|\Phi_{0}\right|<\infty$;
(b) $\mathbb{E} \log ^{+}\left|V_{0}^{1 / 2} \xi_{0}\right|<\infty$;
(c) $\gamma_{1}:=\mathbb{E} \log \left|\Phi_{0}\right|<0$.

(c) using the theorem of Fubini and the hypothesis (1), we obtain:

$$
\gamma_{1}=\mathbb{E} \log \left|\Phi_{0}\right|=\mathbb{E} \int_{0}^{\delta} a\left(X_{u}\right) \mathrm{d} u=\int_{0}^{\delta} \mathbb{E} a\left(X_{u}\right) \mathrm{d} u=\delta \alpha<0 .
$$

(a)

$$
\begin{aligned}
\mathbb{E} \log ^{+}\left|\Phi_{0}\right| & =\mathbb{E} \log ^{+} \exp \int_{0}^{\delta} a\left(X_{u}\right) \mathrm{d} u \leq \mathbb{E} \log ^{+} \exp \int_{0}^{\delta}\left|a\left(X_{u}\right)\right| \mathrm{d} u \\
& =\mathbb{E} \int_{0}^{\delta}\left|a\left(X_{u}\right)\right| \mathrm{d} u=\delta \mathbb{E}\left|a\left(X_{0}\right)\right|<\infty
\end{aligned}
$$

(b) $\mathbb{E} \log ^{+}\left|V_{0}^{1 / 2} \xi_{0}\right| \leq \mathbb{E} \log ^{+} V_{0}^{1 / 2}+\mathbb{E} \log ^{+}\left|\xi_{0}\right|$.

The second term of the upper bound is finite as $\xi_{0}$ is Gaussian. For the first term:

$$
\begin{aligned}
V_{0} & =\int_{0}^{\delta} \exp \left[2 \int_{u}^{\delta} a\left(X_{v}\right) \mathrm{d} v\right] \sigma^{2}\left(X_{u}\right) \mathrm{d} u \leq \int_{0}^{\delta} \exp \left[2 \int_{u}^{\delta}\left|a\left(X_{v}\right)\right| \mathrm{d} v\right] \sigma^{2}\left(X_{u}\right) \mathrm{d} u \\
& \leq \int_{0}^{\delta} \exp \left[2 \int_{0}^{\delta}\left|a\left(X_{v}\right)\right| \mathrm{d} v\right] \sigma^{2}\left(X_{u}\right) \mathrm{d} u=\exp \left[2 \int_{0}^{\delta}\left|a\left(X_{v}\right)\right| \mathrm{d} v\right] \int_{0}^{\delta} \sigma^{2}\left(X_{u}\right) \mathrm{d} u .
\end{aligned}
$$

Thus,

$$
\log ^{+} V_{0} \leq 2 \int_{0}^{\delta}\left|a\left(X_{v}\right)\right| \mathrm{d} v+\log ^{+} \int_{0}^{\delta} \sigma^{2}\left(X_{u}\right) \mathrm{d} u
$$

The first term is of finite expectation according to the hypothesis (1). So does the second term since under the hypothesis (2), the stationarity of $X$ implies that for any $A>0, \mathbb{E}\left[\log ^{+} \int_{0}^{A} \sigma^{2}\left(X_{u}\right) \mathrm{d} u\right]<\infty$. Part (ii). We have for $n \geq 1$,

$$
Y_{n \delta}-\tilde{Y}_{n \delta}=\Phi_{n} \cdots \Phi_{1}\left(Y_{0}-\tilde{Y}_{0}\right)
$$

Under the assumptions and by the ergodic theorem, we have a.s.

$$
\lim _{n} \frac{1}{n} \log \left|\Phi_{n} \cdots \Phi_{1}\right|=\gamma_{1}<0 .
$$

The conclusions immediately follow.

Note that the hypothesis (2) above is automatically satisfied for a bounded function $\sigma$, e.g. if the state space of $X$ is finite.

A consequence of this proposition is that under the law $\mathbb{P}$, if we note $\nu$ the common law of the $\tilde{Y}_{n \delta}$, for any solution $Y^{(\delta)}=\left(Y_{n \delta}\right)$ of equation (4) with arbitrary $Y_{0}, Y_{n \delta}$ converges in law toward $\nu$ when $n \rightarrow \infty$ : $Y^{(\delta)}$ is ergodic. 


\subsection{Ergodicity of the process $\boldsymbol{Y}$}

From now on, we will choose the step size $\delta$ in the dyadic set $\left(2^{-m}\right)$ for integers $m \geq 1$. Under the conditions of Proposition 1, any discretization $Y^{\left(2^{-m}\right)}$ is ergodic. Moreover, as for $m^{\prime} \geq m, Y^{\left(2^{-m^{\prime}}\right)}$ is embedded in $Y^{\left(2^{-m}\right)}$, all these discretizations have the same limit law. This limit law, say $\nu$, should be the one of $Y$ itself if $Y$ was proved to be ergodic. We now prove this by approximating $Y$ by its discretizations.

Proposition 2. Under the conditions of Proposition 1, the linear diffusion $Y$ with the switching $X$ defined by (1) is ergodic.

Proof. Let $\varepsilon>0$ be fixed and choose $A_{\varepsilon}$ such that $\nu\left\{x:|x| \geq A_{\varepsilon}\right\} \leq \varepsilon$. For $\delta=2^{-m}$ and $t>0$, let $n_{t}$ be the largest multiple of $\delta$ lower than $t$. We have $n_{t}<t \leq n_{t}+\delta$. The recursion (2) can be rewritten as

$$
Y_{t}-Y_{n_{t}}=\left[\Phi\left(n_{t}, t\right)-1\right] Y_{n_{t}}+e_{t}
$$

with $e_{t}=V_{n_{t}, t}^{1 / 2} \xi_{n_{t}, t}$. We have:

$$
\mathbb{P}\left(\left|Y_{t}-Y_{n_{t}}\right| \geq 2 \varepsilon\right) \leq \mathbb{P}\left(\left|\left[\Phi\left(n_{t}, t\right)-1\right] Y_{n_{t}}\right| \geq \varepsilon\right)+\mathbb{P}\left[\left|e_{t}\right| \geq \varepsilon\right]
$$

(1) Estimation of $\left|e_{t}\right|$ : we have for $K \geq 0$

$$
\left\{\left|e_{t}\right| \geq \varepsilon\right\}=\left\{\left|e_{t}\right| \geq \varepsilon,\left|\xi_{n_{t}, t}\right| \leq K\right\} \cup\left\{\left|e_{t}\right| \geq \varepsilon,\left|\xi_{n_{t}, t}\right|>K\right\}
$$

As $\xi_{n_{t}, t}$ is standard Gaussian, we fix $K>0$ such that $\mathbb{P}\left(\left|\xi_{n_{t}, t}\right|>K\right) \leq \varepsilon$. Thus

$$
\mathbb{P}\left[\left|e_{t}\right| \geq \varepsilon\right] \leq . \mathbb{P}\left[V_{n_{t}, t}^{1 / 2} \geq \frac{\varepsilon}{K}\right]+\varepsilon .
$$

On the other hand we have similarly to equation (6),

$$
0 \leq V_{n_{t}, t} \leq \exp \left[2 \int_{n_{t}}^{n_{t}+\delta}\left|a\left(X_{v}\right)\right| \mathrm{d} v\right] \int_{n_{t}}^{n_{t}+\delta} \sigma^{2}\left(X_{u}\right) \mathrm{d} u
$$

Thus,

$$
\mathbb{P}\left[V_{n_{t}, t} \geq(\varepsilon / K)^{2}\right] \leq \mathbb{P}\left(\exp \left[2 \int_{n_{t}}^{n_{t}+\delta}\left|a\left(X_{v}\right)\right| \mathrm{d} v\right] \geq 2\right)+\mathbb{P}\left(\int_{n_{t}}^{n_{t}+\delta} \sigma^{2}\left(X_{u}\right) \mathrm{d} u \geq(\varepsilon / K)^{2} / 2\right)
$$

Set $c=\mathbb{E}\left[\left|a\left(X_{0}\right)\right|\right]$. By the Markov inequality, the first term is upper bounded by $2 c \delta / \log (2)$; the second tends to 0 when $\delta \rightarrow 0$ as $\int_{0}^{\delta} \sigma^{2}\left(X_{u}\right) \mathrm{d} u$ tends to zero almost surely (see the remark at the end of Sect. 2). Thus, there exists $\delta_{1}$ such that for any $\delta \leq \delta_{1}$, we have

$$
\mathbb{P}\left[\left|e_{t}\right| \geq \varepsilon\right] \leq 3 \varepsilon
$$

(2) Estimation of the first term: using the fact that $\left|\mathrm{e}^{x}-1\right| \leq \mathrm{e}^{|x|}-1$, we have, for $s>0$,

$$
\begin{aligned}
\mathbb{P}\left[\left|\Phi\left(n_{t}, t\right)-1\right| \geq s\right] & \leq \mathbb{P}\left[\left|\int_{n_{t}}^{t} a\left(X_{u}\right) \mathrm{d} u\right| \geq \log (s+1)\right] \\
& \leq \log (s+1)^{-1} \mathbb{E}\left|\int_{n_{t}}^{t} a\left(X_{u}\right) \mathrm{d} u\right| \leq \log (s+1)^{-1} \mathbb{E} \int_{n_{t}}^{\left(n_{t}+\delta\right)}\left|a\left(X_{u}\right)\right| \mathrm{d} u \\
& =(c \delta) / \log (s+1) .
\end{aligned}
$$


Also, we deduce from the decomposition

$$
\left\{\left|\left[\Phi\left(n_{t}, t\right)-1\right] Y_{n_{t}}\right| \geq \varepsilon\right\}=\left\{\left|\left[\Phi\left(n_{t}, t\right)-1\right] Y_{n_{t}}\right| \geq \varepsilon,\left|Y_{n_{t}}\right|<A_{\varepsilon}\right\} \cup\left\{\left|\left[\Phi\left(n_{t}, t\right)-1\right] Y_{n_{t}}\right| \geq \varepsilon,\left|Y_{n_{t}}\right| \geq A_{\varepsilon}\right\},
$$

that

$$
\begin{aligned}
\mathbb{P}\left[\left|\left[\Phi\left(n_{t}, t\right)-1\right] Y_{n_{t}}\right| \geq \varepsilon\right] & \leq \mathbb{P}\left[\left|\Phi\left(n_{t}, t\right)-1\right| \geq \varepsilon / A_{\varepsilon}\right]+\mathbb{P}\left[\left|Y_{n_{t}}\right| \geq A_{\varepsilon}\right] \\
& \leq(c \delta) / \log \left(\left(\varepsilon / A_{\varepsilon}\right)+1\right)+\mathbb{P}\left[\left|Y_{n_{t}}\right| \geq A_{\varepsilon}\right]
\end{aligned}
$$

Choose a $\delta$ such that $\delta \leq \delta_{1}$ and $c \delta / \log \left(\left(\varepsilon / A_{\varepsilon}\right)+1\right)<\varepsilon$. With this $\delta$, we have

$$
\mathbb{P}\left[\left|\left[\Phi\left(n_{t}, t\right)-1\right] Y_{n_{t}}\right| \geq \varepsilon\right] \leq \mathbb{P}\left[\left|Y_{n_{t}}\right| \geq A_{\varepsilon}\right]+\varepsilon
$$

(3) End of the proof: summarizing from the estimations (8-10): we obtain $\forall \varepsilon>0, \exists A_{\varepsilon}, \exists \delta, \forall t>0, \exists n_{t}$, such that $\nu\left\{|x| \geq A_{\varepsilon}\right\} \leq \varepsilon, n_{t}<t \leq n_{t}+\delta$ and

$$
\mathbb{P}\left(\left|Y_{t}-Y_{n_{t}}\right| \geq 2 \varepsilon\right) \leq \mathbb{P}\left[\left|Y_{n_{t}}\right| \geq A_{\varepsilon}\right]+4 \varepsilon
$$

Now consider a sequence $\left(Y_{t_{k}}\right)_{k}$ with $t_{k} \rightarrow \infty$. The previous inequality for $t=t_{k}$ gives:

$$
\mathbb{P}\left(\left|Y_{t_{k}}-Y_{n_{t_{k}}}\right| \geq 2 \varepsilon\right) \leq \mathbb{P}\left[\left|Y_{n_{t_{k}}}\right| \geq A_{\varepsilon}\right]+4 \varepsilon
$$

Thus,

$$
\limsup _{k \rightarrow \infty} \mathbb{P}\left(\left|Y_{t_{k}}-Y_{n_{t_{k}}}\right| \geq 2 \varepsilon\right) \leq \nu\left\{|x| \geq A_{\varepsilon}\right\}+4 \varepsilon \leq 5 \varepsilon
$$

Let $C(\nu)$ be the set of continuity points of the distribution function $F_{\nu}$ of the law $\nu$. Let $x \in C(\nu)$, and choose $\varepsilon>0$ such that $x \pm 2 \varepsilon \in C(\nu)$. We have

$$
\mathbb{P}\left(Y_{t_{k}} \leq x\right) \leq \mathbb{P}\left(Y_{n_{t_{k}}} \leq x+2 \varepsilon\right)+\mathbb{P}\left(\left|Y_{t_{k}}-Y_{n_{t_{k}}}\right| \geq 2 \varepsilon\right)
$$

and in a similar manner

$$
\mathbb{P}\left(Y_{n_{t_{k}}} \leq x-2 \varepsilon\right) \leq \mathbb{P}\left(Y_{t_{k}} \leq x\right)+\mathbb{P}\left(\left|Y_{t_{k}}-Y_{n_{t_{k}}}\right| \geq 2 \varepsilon\right)
$$

Thus

$$
F_{\nu}(x-2 \varepsilon)-5 \varepsilon \leq \liminf _{k} \mathbb{P}\left(Y_{t_{k}} \leq x\right) \leq \limsup _{k} \mathbb{P}\left(Y_{t_{k}} \leq x\right) \leq F_{\nu}(x+2 \varepsilon)+5 \varepsilon .
$$

Letting $\varepsilon$ go to 0 (with $x \pm 2 \varepsilon \in C(\nu)$, which is possible since $C(\nu)$ is dense as the complementary of a countable set), we obtain:

$$
\lim _{k} \mathbb{P}\left(Y_{t_{k}} \leq x\right)=F_{\nu}(x), \quad x \in C(\nu)
$$

\section{Linear Diffusion With Finite MARKOV SWITCHING}

In this section, we examine the particular case where the process $X$ is a Markov jump process with a finite state space $E=\{1,2, \cdots, N\}, N>1$ (cf. Feller [5], Coccoza [4], Chap. 8). We assume that the intensity function $\lambda$ of $X$ is positive and the jump kernel $q(x, y)$ on $E$ is irreducible and satisfies $q(x, x)=0$, for each $x \in E$. The process $X$ is then a ergodic Markov process and we denote its invariant probability measure by $\mu$. 
Let $\left(P_{t}\right)$ be the associated Markov semi-group. Let us recall the following basic property of $X$. For small positive $h$ and every $x \in E$,

$$
P_{h}(x, y)= \begin{cases}\lambda(x) h q(x, y)+o(h), & y \neq x \\ 1-\lambda(x) h+o(h), & y=x\end{cases}
$$

To fix the notations, we consider the canonical version $\left(\Omega, \mathcal{A},\left(Q_{x}\right)_{x \in E}\right)$ of $X$ where $\Omega=D([0, \infty[)$ is the space of the real càdlàg functions on $[0, \infty[$ and $\mathcal{A}$ the $\sigma$-algebra associated to the Skohokod metric. The product probabilities will be denoted $\mathbb{P}_{x}=Q_{x} \otimes Q^{\prime}$. Particularly, under the probability $\mathbb{P}_{\mu}=Q_{\mu} \otimes Q^{\prime}$, the process $X$ is stationary.

The transcription of Proposition 2 in the present case gives:

Corollary 1. Assume that the Markovian switching process $X$ with finite number of states is stationary with invariant distribution $\mu$. Then the diffusion of O.U. Y with Markovian switching $X$ is ergodic as soon as:

$$
\alpha=\sum_{x \in E} a(x) \mu(x)<0
$$

Next we search for sufficient conditions that guarantee moments for the stationary distribution of the diffusion process. We will see in Paragraph 3.2 that we recover the results of Basak et al. [1] for this particular problem. However our proof is different.

\subsection{Existence of moments for the stationary distribution $\nu$ of $Y$}

We prove the following result.

Proposition 3. Let $s>0$. Assume that there is a positive function $\psi$ on $E$ such that

$$
\left(\mathbf{C}_{\mathbf{s}}\right) \quad[s a(x)-\lambda(x)] \psi(x)+\lambda(x) \sum_{y \neq x} q(x, y) \psi(y)<0, \quad x \in E
$$

Then the stationary distribution $\nu$ of $Y$ has a moment of order $s$.

Proof. Under the assumed condition, we claim that we can find a $\delta>0$ such that for the associated discretization $Y^{(\delta)}$, the series representing the stationary solution $\tilde{Y}_{n \delta}$ in equation (5) converges absolutely in $L^{s}$. The main step is to prove that for some constants $C$ and $0 \leq \rho<1$,

$$
\mathbb{E}_{\mu}\left[\left(\Phi_{1} \cdots \Phi_{k}\right)^{s}\right] \leq C \rho^{k}
$$

Assume for the moment that (13) is true. First let us prove that $Y$ is ergodic via Corollary 1 . Indeed we have then

$$
\frac{1}{k} \mathbb{E}_{\mu} \log \left[\left(\Phi_{1} \cdots \Phi_{k}\right)^{s}\right] \leq \frac{1}{k} \log \mathbb{E}_{\mu}\left[\left(\Phi_{1} \cdots \Phi_{k}\right)^{s}\right] \leq \frac{1}{k} \log C+\log \rho .
$$

Letting $k \rightarrow \infty$ and by noticing that $\frac{1}{k} \mathbb{E}_{\mu} \log \left[\left(\Phi_{1} \cdots \Phi_{k}\right)^{s}\right]=\delta s \alpha$ proves that $\alpha<0$ and that the diffusion $Y$ is ergodic.

Secondly, for any function $f$ on $E$ define $\|f\|_{\infty}=\sup _{x}|f(x)|$. Starting from (5), we have for $s \geq 1$

$$
\left(\mathbb{E}_{\mu}\left[\left|\tilde{Y}_{n \delta}\right|^{s}\right]\right)^{1 / s} \leq \sum_{k=0}^{\infty}\left(\mathbb{E}_{\mu}\left|\Phi_{n} \Phi_{n-1} \cdots \Phi_{n-k+1} V_{n-k}^{1 / 2} \xi_{n-k}\right|^{s}\right)^{1 / s}
$$


and for $0<s \leq 1$,

$$
\mathbb{E}_{\mu}\left[\left|\tilde{Y}_{n \delta}\right|^{s}\right] \leq \sum_{k=0}^{\infty} \mathbb{E}_{\mu}\left[\left|\Phi_{n} \Phi_{n-1} \cdots \Phi_{n-k+1} V_{n-k}^{1 / 2} \xi_{n-k}\right|^{s}\right] .
$$

By Independence of the Gaussian variable $\xi_{n-k}$ and noticing that the $V_{i}$ 's are bounded by

$$
\left|V_{i}\right| \leq \delta\left\|\sigma^{2}\right\|_{\infty} \mathrm{e}^{2 \delta\|a\|_{\infty}}
$$

these series are upper-bounded by a converging geometric series following the claim (13). So the stationary distribution $\nu$ of the diffusion $Y$, which is also the law of $\tilde{Y}_{n \delta}$ has the moment of order $s$.

We now prove the main claim (13). First fix an arbitrary $\delta>0$ and defined the operator $A$ by

$$
A \varphi(x)=\mathbb{E}_{x}\left[\left|\Phi_{1}\right|^{s} \varphi\left(X_{\delta}\right)\right], \quad x \in E,
$$

for any function $\varphi$ on $E$. In particular $A \mathbb{1}=\mathbb{E}_{x}\left[\left|\Phi_{1}\right|^{s}\right]$ where $\mathbb{1}$ is the function taking the constant value 1 . Let be the sigma-algebra $\mathcal{F}_{k}=\sigma\left(X_{t}, t \leq k \delta\right)$. Then by Markov property and successive conditioning,

$$
\begin{aligned}
\mathbb{E}_{x}\left[\left|\Phi_{1} \cdots \Phi_{k}\right|^{s}\right] & =\mathbb{E}_{x}\left[\left|\Phi_{1} \cdots \Phi_{k-1}\right|^{s} \mathbb{E}_{x}\left(\left|\Phi_{k}\right|^{s} \mid \mathcal{F}_{k-1}\right)\right] \\
& =\mathbb{E}_{x}\left[\left|\Phi_{1} \cdots \Phi_{k-1}\right|^{s} A \mathbb{1}\left(X_{(k-1) \delta}\right)\right] \\
& =A^{k} \mathbb{1}(x) .
\end{aligned}
$$

It follows that $\mathbb{E}_{\mu}\left[\left|\Phi_{1} \cdots \Phi_{k}\right|^{s}\right]=\sum_{x} A^{k} \mathbb{1}(x) \mu(x)$. The claim will be proved if we can choose $\delta$ such that the spectral radius of the operator $A$ is smaller than 1 .

We now compute precisely $A$. Let $N_{*}$ be the number of jumps on the interval $[0, \delta]$. We have for small $\delta$,

$$
\mathbb{E}_{x} \mathbb{1}_{N_{*}=0}=1-\lambda(x) \delta+o(\delta), \quad \mathbb{E}_{x} \mathbb{1}_{N_{*}=1}=\lambda(x) \delta+o(\delta), \quad \mathbb{E}_{x} \mathbb{1}_{N_{*}>1}=o(\delta) .
$$

Therefore,

$$
\begin{aligned}
& \mathbb{E}_{x}\left[\left|\Phi_{1}\right|^{s} \varphi\left(X_{\delta}\right) \mathbb{1}_{N_{*}>1}\right]=o(\delta), \\
& \mathbb{E}_{x}\left[\left|\Phi_{1}\right|^{s} \varphi\left(X_{\delta}\right) \mathbb{1}_{N_{*}=0}\right]=[1-\lambda(x) \delta] \mathrm{e}^{\delta s a(x)} \varphi(x)+o(\delta)=\{1+\delta[s a(x)-\lambda(x)]\} \varphi(x)+o(\delta) .
\end{aligned}
$$

To compute the remaining term, note that the probability density that there is exactly one jump at time $u \in[0, \delta]$ and from $x$ to $y \neq x$ is

$$
\lambda(x) \mathrm{e}^{-\lambda(x) u} q(x, y) \mathrm{e}^{-\lambda(y)[\delta-u]} .
$$

Consequently

$$
\begin{aligned}
\mathbb{E}_{x}\left[\left|\Phi_{1}\right|^{s} \varphi\left(X_{\delta}\right) \mathbb{1}_{N_{*}=1}\right] & =\int_{0}^{\delta} \sum_{y \neq x}\left\{\left[\lambda(x) \mathrm{e}^{-\lambda(x) u} q(x, y) \mathrm{e}^{-\lambda(y)[\delta-u]}\right] \mathrm{e}^{s[a(x) u+a(y)(\delta-u)]} \varphi(y)\right\} \mathrm{d} u+o(\delta) \\
& =\sum_{y \neq x} \lambda(x) q(x, y) \varphi(y) \frac{\mathrm{e}^{\delta[s a(x)-\lambda(x)]}-\mathrm{e}^{\delta[s a(y)-\lambda(y)]}}{\lambda(y)-\lambda(x)+s[a(x)-a(y)]}+o(\delta) \\
& =\delta \lambda(x) \sum_{y \neq x} q(x, y) \varphi(y)+o(\delta) .
\end{aligned}
$$


Summarizing we have proved that

$$
A \varphi(x)=\{1+\delta[s a(x)-\lambda(x)]\} \varphi(x)+\delta \lambda(x) \sum_{y \neq x} q(x, y) \varphi(y)+o(\delta) .
$$

Under the condition $\left(\mathbf{C}_{\mathbf{s}}\right)$, we can then take a sufficient small $\delta$ such that for the positive function $\psi$ on $E$, we have $0 \leq A \psi<\psi$. Note that here $A$ is nonnegative and irreducible. Therefore according to the theorem of Perron-Frobenus (see for example [11], p. 492), the existence of such a $\psi>0$ is equivalent to the fact that the spectral radius of $A$ is lower than 1 .

The proof of the proposition is complete.

We conclude this subsection by pointing out that the condition $\left(\mathbf{C}_{\mathbf{s}}\right)$ above is equivalent to the following two conditions:

(e1) $\forall x \in E: s a(x)-\lambda(x)<0$;

(e2) the spectral radius of the matrix $\quad M_{s}=\left(q(x, y) \frac{\lambda(x)}{\lambda(x)-s a(x)}\right), \quad x, y \in E \quad$ is smaller than 1.

Indeed, when $\left(\mathbf{C}_{\mathbf{s}}\right)$ is satisfied, necessarily Condition (e1) is verified. Moreover as, for each $x \operatorname{sa}(x)-\lambda(x) \neq 0$, the inequalities in $\left(\mathbf{C}_{\mathbf{s}}\right)$ can be written in matrix form as $0 \leq M_{s} \psi<\psi$. Therefore the spectral radius of $M_{s}$ is lower than 1 .

Conversely, conditions (e1) and (e2) imply Condition $\left(\mathbf{C}_{\mathbf{s}}\right)$ according to these same reasons.

\subsection{Comparison with the results of [1]}

As mentioned we compare our result on the moments to the one given in [1]. Let $\Lambda$ be the infinitesimal generator of $X$ :

$$
\Lambda(i, j)= \begin{cases}\lambda(i) q(i, j), & \text { si } i \neq j \\ -\lambda(i), & \text { si } i=j .\end{cases}
$$

The authors of [1] consider a vector-valued diffusion $Y \in \mathbb{R}^{d}$ solution of (1), with matrix-valued coefficients $(a(i), \sigma(i)), i=1, \ldots, N$. They use the following condition

(A2) There exist $N$ symmetric positive definite $d \times d$ matrices $B_{i}, \gamma>0, s>0$ such that:

$$
u^{\prime} B_{i} a(i) u+\frac{1}{s} u^{\prime} B_{i} u \sum_{j=1}^{N} \Lambda_{i j}\left(\frac{u^{\prime} B_{j} u}{u^{\prime} B_{i} u}\right)^{s / 2} \leq-\gamma|u|^{2}, \quad \forall u \in \mathbb{R}^{d}, u \neq 0, i=1, \ldots, N
$$

Then the authors proved that (see their Th. 3.1 and Lem. 3.2), under the condition (A2), the process $\left(X_{t}, Y_{t}\right)$ is ergodic and the limit law of $Y_{t}$ has a moment of order $s$.

Let us show that (A2), when particularized to the univariate case $d=1$, is equivalent to our Condition $\left(\mathbf{C}_{\mathbf{s}}\right)$ given in Proposition 3. Substituting $\lambda$ and $q$ for $\Lambda$ in (A2) gives

$$
(s a(i)-\lambda(i)) B_{i}^{\frac{s}{2}}+\sum_{j \neq i} \lambda(i) q(i, j) B_{j}^{\frac{s}{2}} \leq-s \gamma B_{i}^{\frac{s}{2}-1}, \quad i=1, \ldots, N
$$

Clearly this implies $\left(\mathbf{C}_{\mathbf{s}}\right)$. On the other hand, under $\left(\mathbf{C}_{\mathbf{s}}\right)$, there is a $h>0$ such that

$$
(s a(i)-\lambda(i)) \psi_{i}+\sum_{j: j \neq i} \lambda(i) q(i, j) \psi_{j} \leq-h, \quad i=1, \ldots N
$$

Set $B_{i}=\psi_{i}^{-\frac{s}{2}}$. If we take a $\gamma$ such that for all $i,-h \leq-s \gamma B_{i}^{\frac{s}{2}-1}$, the above inequality is nothing else but (A2). 


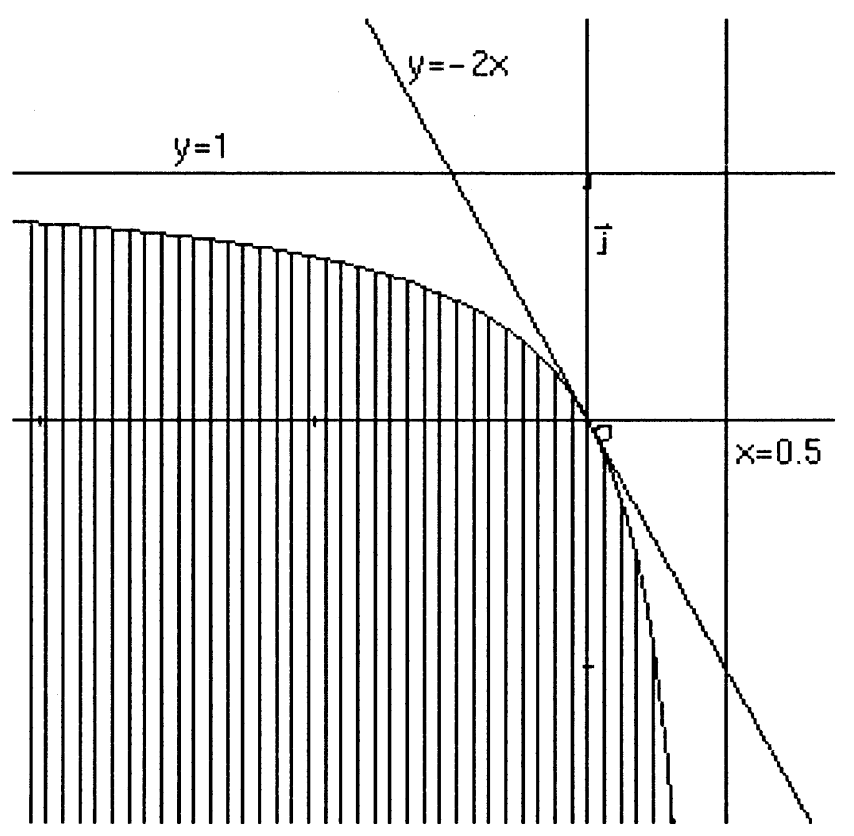

Figure 1. Linear diffusion with two Markov regimes $(\alpha=1, \beta=2)$ : the ergodicity area $(\mathbf{E})$ is under the line of equation $y=-2 x$; the second-order stability area $(\mathbf{E} 2)$ is hashed.

\subsection{Example: A linear diffusion with two regimes}

We conclude the paper with an illustrative example where $X$ is a Markov jump process with two states $E=\{1,2\}$, an intensity function $\alpha=\lambda(1)>0, \beta=\lambda(2)>0$. Then the switching transition matrix is $q=\left(\begin{array}{ll}0 & 1 \\ 1 & 0\end{array}\right)$ and the invariant law of $X$ is $\mu=(\beta, \alpha) /(\alpha+\beta)$. We then obtain:

- ergodicity of $Y$ when:

$$
(\mathbf{E}): \quad \alpha a(2)+\beta a(1)<0 ;
$$

- ergodicity and existence of a moment of order $s$ for $Y$ :

$$
\text { (E2) }:\left\{\begin{aligned}
(i) & s a(1)-\alpha<0, \quad s a(2)-\beta<0 \\
(i i) & a(1) \beta+a(2) \alpha-s a(1) a(2)<0 .
\end{aligned}\right.
$$

Figure 1 displays these regions $(\mathbf{E})$ and $(\mathbf{E 2})$ in the case $\alpha=1, \beta=2$ (the axes are named $(x, y)$ for $(a(1), a(2))$. In Figure 2, we display a simulated path of such a diffusion $Y$ with the following parameters: $\alpha=1, \beta=2$, $a(1)=-1, a(2)=1$ and $\sigma(1)=\sigma(2)=1$. In this case we have shown that the diffusion is ergodic and has moments for any $s<1$. The diffusion switches between the explosive regime $2\left(a(2)=1\right.$ with probability $\left.\frac{1}{3}\right)$ and the stable regime $1\left(a(1)=-1\right.$ with probability $\left.\frac{2}{3}\right)$. Note that the variance of the stationary Ornstein-Uhlenbeck process with drift $a=\mathbb{E}\left(a\left(X_{u}\right)\right)=-\frac{1}{3}$ is $\sigma_{s t}^{2}=\frac{3}{2}$.

Acknowledgements. The authors thank the referees for their important comments, particularly for a major improvement in Section 3. 


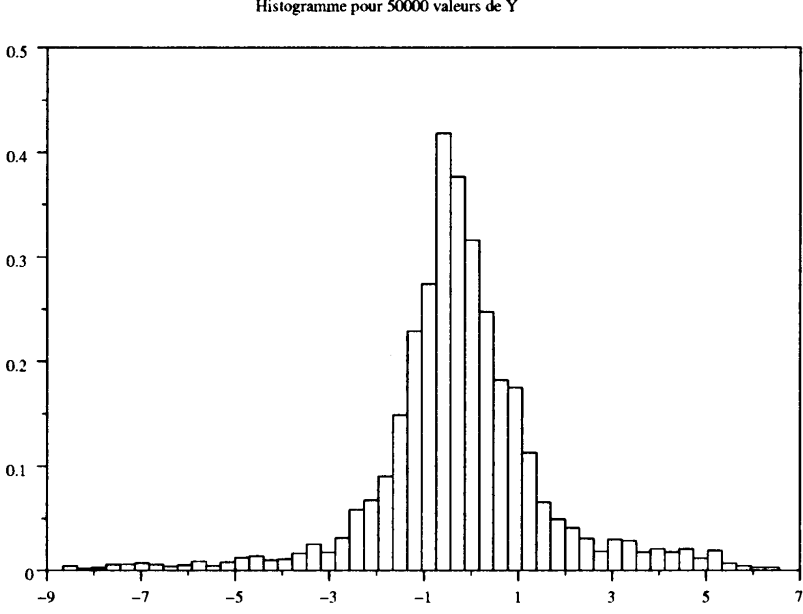

(a)

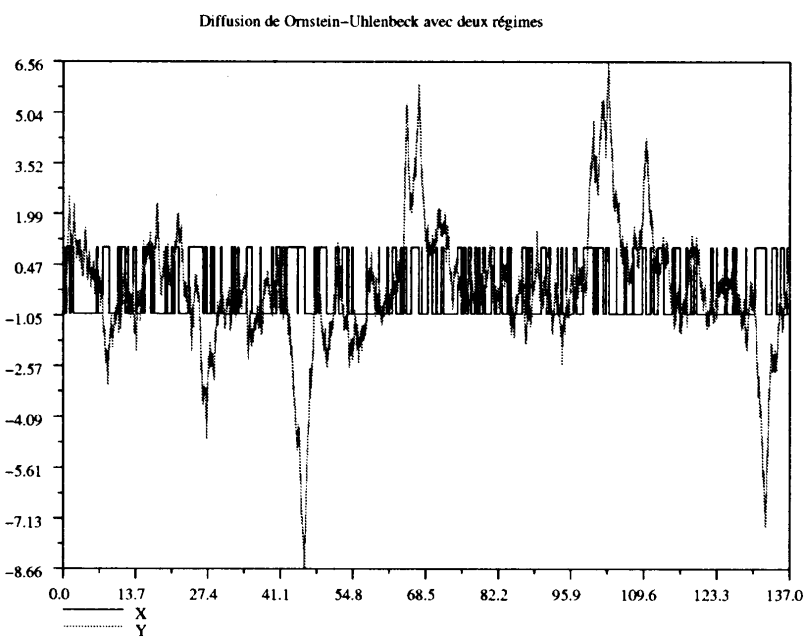

(b)

Figure 2. (a) Simulation of a diffusion with two regimes and parameters $\alpha=1, \beta=2$, $a(1)=-1, a(2)=1, \sigma(1)=\sigma(2)=1$. (b) Histogram of the diffusion for 50000 values regularly sampled.

\section{REFERENCES}

[1] G.K. Basak, A. Bisi and M.K. Ghosh, Stability of random diffusion with linear drift. J. Math. Anal. Appl. 202 (1996) 604-622.

[2] P. Bougerol and N. Picard, Strict stationarity of generalized autoregressive processe. Ann. Probab. 20 (1992) 1714-1730.

[3] A. Brandt, The stochastic equation $Y_{n+1}=A_{n} Y_{n}+B_{n}$ with stationnary coefficients. Adv. Appl. Probab. 18 (1986) 211-220.

[4] C. Cocozza-Thivent, Processus stochastiques et fiabilité des systèmes. Springer (1997).

[5] W. Feller, An Introduction to Probability Theory, Vol. II. Wiley (1966).

[6] C. Francq and M. Roussignol, Ergodicity of autoregressive processes with Markov switching and consistency of maximumlikelihood estimator. Statistics 32 (1998) 151-173.

[7] J.D. Hamilton, A new approach to the economic analysis of nonstationary time series and the business cycle. Econometrica 57 (1989) 151-173.

[8] J.D. Hamilton, Analysis of time series subject to changes in regime. J. Econometrics 45 (1990) 39-70.

[9] J.D. Hamilton, Specification testing in Markov-switching time series models. J. Econometrics 70 (1996) 127-157.

[10] B. Hansen, The likelihood ratio test under nonstandard conditions: Testing the Markov switching model of GNP. J. Appl. Econometrics 7 (1996) 61-82.

[11] R.A. Horn and C.R. Johnson, Matrix Analysis. Cambridge University Press (1985).

[12] Y. Ji and H.J. Chizeck, Controllability, stabilizability and continuous-time Markovian jump linear quadratic control. IEEE Trans. Automat. Control 35 (1990) 777-788.

[13] I. Karatzas and S.E. Shreve, Brownian Motion and Stochastic calculus, 2nd Ed. Springer, New York (1991).

[14] M. Mariton, Jump linear systems in Automatic Control. Dekker (1990).

[15] R.E. McCullogh and R.S. Tsay, Statistical analysis of econometric times series via Markov switching models, J. Time Ser. Anal. 15 (1994) 523-539.

[16] B. Øksendal, Stochastic Differential Equations, 5th Ed. Springer-Verlag, Berlin (1998).

[17] J.F. Yao and J.G. Attali, On stability of nonlinear AR processes with Markov switching. Adv. Appl. Probab. 32 (2000) $394-407$. 